\title{
DENSITIES OF SETS OF INTEGERS AND TRANSFORMS OF SEQUENCES OF ZEROS AND ONES
}

BY

\section{RALPH PALMER AGNEW}

1. Introduction. It is our purpose to obtain formulas and tables involving several standard methods for determining densities of sets of positive integers. These methods fall within a general class of methods defined in $\$ 3$. Theorem 3.3 and its applications, including four tables, constitute our main results.

Let $E$ be an infinite set of positive integers which may be denoted, in increasing order, by $\lambda_{1}, \lambda_{2}, \lambda_{3}, \cdots$. Let $s_{1}, s_{2}, s_{3}, \cdots$, the characteristic sequence of the set $E$, be defined by the condition that $s_{n}=0$ if $n$ is not in $E$ and $s_{n}=1$ if $n$ is in $E$. The counting function $S(t)$ of $E$ is defined for each real $t$ by the formula

$$
S(t)=\sum_{k \leq t} s_{k}=\sum_{\lambda_{k} \leq t} 1=\sum_{k \leq t, k \in E} 1 .
$$

Thus $S(t)$ is, for each $t>0$, the number of elements of $E$ in the closed interval $(0, t)$ and the function $D(t)$ defined for $t>0$ by

$$
D(t)=S(t) / t
$$

is, for each $t>0$, a partial density of $E$, that is, the density in $(0, t)$ of the part of $E$ in $(0, t)$. The upper density $\bar{D}$ of $E$ is defined by

$$
\bar{D}=\limsup _{n \rightarrow \infty} D(n)=\limsup _{t \rightarrow \infty} D(t) .
$$

In case $\lim D(t)$ exists, this limit is denoted by $D$ and is called the density of E.

Other determinations of densities and upper densities are obtained by applying summability transformations to the sequence $D(n)$ or function $D(t)$ of partial densities. Many considerations are simplified by the fact that $D(t)=0$ when $t<1, D(t)$ is continuous except for jumps at the integers in $E$, and $0 \leqq D(t) \leqq 1$. For example, applying the arithmetic mean (or $C_{1}$ ) transformation $M$ gives the upper mean (or arithmetic mean) density $\bar{D}_{M}$ defined by

$$
\bar{D}_{M}=\limsup _{n \rightarrow \infty} \frac{1}{n} \sum_{k=1}^{n} D(k)=\limsup _{x \rightarrow \infty} \frac{1}{x} \int_{0}^{x} D(t) d t .
$$

It is easy to show that the last two members of (1.4) are equal. Putting

Received by the editors July 11, 1956. 


$$
H_{1}(x)=\frac{1}{x} \int_{0}^{x} D(t) d t
$$

and defining $H_{2}(x), H_{3}(x), \cdots$ by

$$
H_{r}(x)=\frac{1}{x} \int_{0}^{x} H_{r-1}(t) d t, \quad r=2,3, \cdots,
$$

we find, by changes of order of integration, the familiar formulas

$$
H_{r}(x)=\frac{1}{(r-1) ! x} \int_{0}^{x}\left(\log \frac{x}{t}\right)^{r-1} D(t) d t .
$$

Accordingly we define the Hölder upper mean density $\bar{D}_{M}^{(r)}$ of order $r$ by the formula

$$
\bar{D}_{M}^{(r)}=\limsup _{x \rightarrow \infty} \frac{1}{(r-1) ! x} \int_{0}^{x}\left(\log \frac{x}{t}\right)^{r-1} D(t) d t .
$$

Comparing (1.4) and (1.44), we see that $\bar{D}_{M}=\bar{D}_{M}^{(1)}$.

Similarly, application of the Abel power series transformation $A$ to the sequence $D(n)$ gives the upper Abel density $\bar{D}_{A}$ defined by

$$
\bar{D}_{A}=\limsup _{r \rightarrow 1}(1-r) \sum_{k=1}^{\infty} r^{k} D(k) .
$$

Putting $r=e^{-1 / x}$ and letting $F_{A}(x)$ denote the last part of (1.5) gives

$$
F_{A}(x)=\left(1-e^{-1 / x}\right) \sum_{k=1}^{\infty} \int_{k}^{k+1} e^{-k / x} D(k) d t
$$

and it is not difficult to show that, as $x \rightarrow \infty$,

$$
F_{A}(x)=o(1)+\frac{1}{x} \int_{0}^{\infty} e^{-t / x} D(t) d t .
$$

Thus we obtain the formula

$$
\bar{D}_{A}=\limsup _{x \rightarrow \infty} \frac{1}{x} \int_{0}^{\infty} e^{-t / x} D(t) d t
$$

which is more convenient than (1.5).

Application to $D(t)$ of a transformation employed by Poisson gives the upper Poisson density $\bar{D}_{P}$ defined by

$$
\bar{D}_{P}=\limsup _{x \rightarrow \infty} \frac{2}{\pi} \int_{0}^{\infty} \frac{x}{t^{2}+x^{2}} D(t) d t .
$$

Some known and useful formulas involving $\bar{D}_{P}$ are easily obtained in terms 
of functions $D_{P}(x)$ and $L(t)$ defined by

$$
D_{P}(x)=\frac{2}{\pi} \int_{0}^{\infty} \frac{x}{t^{2}+x^{2}} D(t) d t, \quad L(t)=\log \left(1+\frac{x^{2}}{t^{2}}\right) .
$$

Starting with (1.61) gives, for each $x>0$,

$$
\begin{aligned}
D_{P}(x) & =\frac{1}{\pi x} \sum_{n=1}^{\infty} \int_{n}^{n+1} \frac{2 x^{2}}{t^{2}+x^{2}} \frac{S(n)}{t} d t \\
& =\frac{1}{\pi x} \sum_{n=1}^{\infty}[L(n)-L(n+1)] S(n) \\
& =\frac{1}{\pi x} \sum_{n=1}^{\infty} L(n)[S(n)-S(n-1)] \\
& =\frac{1}{\pi x} \sum_{n=1}^{\infty} L(n) s_{n}=\frac{1}{\pi x} \sum_{n=1}^{\infty} L\left(\lambda_{n}\right) \\
& =\frac{1}{\pi x} \sum_{n=1}^{\infty} \log \left(1+\frac{x^{2}}{\lambda_{n}^{2}}\right)=\frac{1}{\pi x} \log \prod_{n=1}^{\infty}\left(1+\frac{x^{2}}{\lambda_{n}^{2}}\right)
\end{aligned}
$$

and hence

$$
\bar{D}_{P}=\limsup _{x \rightarrow \infty} \frac{1}{\pi x} \log \prod_{n=1}^{\infty}\left(1+\frac{x^{2}}{\lambda_{n}^{2}}\right) .
$$

The remaining upper density $\bar{D}_{a}$ which we treat falls within the above category but does not originate within the above category. It is obtained by applying the Abel transformation to the characteristic sequence $s_{n}$ (instead of to the partial densities) and is defined by

$$
\bar{D}_{a}=\limsup _{r \rightarrow 1}(1-r) \sum_{k=1}^{\infty} r^{k} s_{k} .
$$

Since (1.1) and (1.2) imply that $D(n)=\left(s_{1}+\cdots+s_{n}\right) / n$ and hence that $s_{k}=k D(k)-(k-1) D(k-1),(1.7)$ can be put in the form

$$
\bar{D}_{a}=\limsup _{r \rightarrow 1}(1-r)^{2} \sum_{k=1}^{\infty} k r^{k} D(k)
$$

where a transform of $D(k)$ is involved. Perhaps the simplest way to obtain alternative useful formulas for $\bar{D}_{a}$ is to start by writing (1.7) in the form

$$
\bar{D}_{a}=\limsup _{r \rightarrow 1}(1-r) \int_{0}^{\infty} r^{t} d s(t) .
$$

Writing $r^{t}$ in the form $\exp \left(-t \log r^{-1}\right)$ and putting $x^{-1}=\log r^{-1}$ then gives 


$$
\bar{D}_{a}=\limsup _{x \rightarrow \infty} \frac{1}{x} \int_{0}^{\infty} e^{-t / x} d S(t)
$$

and integration by parts gives

$$
\bar{D}_{a}=\limsup _{x \rightarrow \infty} \frac{1}{x^{2}} \int_{0}^{\infty} e^{-t / x} S(t) d t
$$

and hence

$$
\bar{D}_{a}=\limsup _{x \rightarrow \infty} \frac{1}{x} \int_{0}^{\infty} \frac{t}{x} e^{-t / x} D(t) d t .
$$

While our principal notations and terminologies are given in terms of densities of sets of integers, it is easy to modify the terminologies and notations and to view our work as being a study of transforms of sequences of zeros and ones. Viewing the characteristic sequence $s_{1}, s_{2}, \cdots$ (instead of the set $E$ of integers) as being the fundamental entity upon which we operate, let $\bar{S}=\lim \sup _{n \rightarrow \infty} s_{n}$. The sequence $D(1), D(2), \ldots$ is the sequence of arithmetic means of the sequence $s_{n}$ and we denote its superior limit by $\bar{M}$ or $\bar{M}^{(1)}$ so that $\bar{D}=\bar{M}=\bar{M}^{(1)}$. Similarly the mean density $\bar{D}_{M}$ is the superior limit of the arithmetic mean transform of the arithmetic mean transform of the sequence $s_{n}$ and we denote this by $\bar{M}^{(2)}$ so that $\bar{D}_{M}=\bar{M}^{(2)}$. Likewise, for each $r \geqq 1, \bar{D}_{M}^{(r)}$ is the superior limit of the iterate of order $(r+1)$ of the arithmetic mean transform of $s_{n}$ and we denote this by $\bar{M}^{(r+1)}$ so that $\bar{D}_{M}^{(r)}=\bar{M}^{(r+1)}$. As (1.5) shows, $\bar{D}_{A}$ is the superior limit of the Abel transform of the arithmetic mean transform of $s_{n}$ and we denote this by $\bar{A} \bar{M}$ so that $\bar{D}_{A}=\bar{A} \bar{M}$. As (1.6) shows, $\bar{D}_{P}$ is the superior limit of the Poisson transform of the (integral) arithmetic mean transform of the function $s(t)$ associated with the sequence $s_{n}$ and we denote this by $\bar{P} \bar{M}$ so that $\bar{D}_{P}=\bar{P} \bar{M}$. Finally, (1.7) shows that $\bar{D}_{a}$ is the superior limit of the Abel transform of the sequence $s_{n}$ and we denote this by $\bar{A}$ so that $\bar{D}_{a}=\bar{A}$.

2. Alternative formulas for the densities. Changing the variables of integration from $t$ to $x t$ in (1.44), (1.53), (1.75) and (1.6) gives

$$
\bar{D}_{M}^{(r)}=\limsup _{x \rightarrow \infty} \frac{1}{(r-1) !} \int_{0}^{1}\left(\log \frac{1}{t}\right)^{r-1} D(x t) d t,
$$

$$
\bar{D}_{A}=\limsup _{x \rightarrow \infty} \int_{0}^{\infty} e^{-t} D(x t) d t
$$

$$
\bar{D}_{a}=\limsup _{x \rightarrow \infty} \int_{0}^{\infty} t e^{-t} D(x t) d t
$$

$$
\bar{D}_{P}=\limsup _{x \rightarrow \infty} \frac{2}{\pi} \int_{0}^{\infty} \frac{1}{1+t^{2}} D(x t) d t .
$$


Our next formulas involve the well known and tabulated exponential integral function $E(t)$ defined by

$$
E(t)=\int_{t}^{\infty} \frac{e^{-u}}{u} d u,
$$

the function $E(t)$ is frequently tabulated under the guise $-E i(-t)$. Replacing $D(x t)$ by $S(x t) / x t$ in (2.01), (2.02), (2.03) and (2.04) and integrating by parts gives

$$
\begin{aligned}
\bar{D}_{M}^{(r)} & =\limsup _{x \rightarrow \infty} \frac{1}{r ! x} \int_{0}^{1}\left(\log \frac{1}{t}\right)^{r} d S(x t), \\
\bar{D}_{A} & =\limsup _{x \rightarrow \infty} \frac{1}{x} \int_{0}^{\infty} E(t) d S(x t), \\
\bar{D}_{a} & =\limsup _{x \rightarrow \infty} \frac{1}{x} \int_{0}^{\infty} e^{-t} d S(x t), \\
\bar{D}_{P} & =\limsup _{x \rightarrow \infty} \frac{1}{\pi x} \int_{0}^{\infty} \log \left(1+\frac{1}{t^{2}}\right) d S(x t) .
\end{aligned}
$$

3. A general class of densities. Each of (2.21), (2.22), (2.23) and (2.24) involves a transformation of the form

$$
F(x)=\frac{1}{x} \int_{0}^{\infty} \phi(t) d S(x t) .
$$

In the case of (2.21), we have $\phi(t)=0$ when $t \geqq 1$. In each case $\phi(t) \geqq 0, \phi(t)$ is continuous and monotone decreasing over $t>0$, and $\int_{0}^{\infty} \phi(t) d t=1$. We assume henceforth that $\phi(t)$ has these properties and, in accordance with notation used above, define an upper density $\bar{D}_{\phi}$ by the formula

$$
\bar{D}_{\phi}=\limsup _{x \rightarrow \infty} F(x) \text {. }
$$

In addition to (3.1), a useful formula for $F(x)$ is obtained by integration by parts. For each $x>0$ we have $S(x t)=x t D(x t)$ and hence $\phi(t) S(x t)$ $=x t \phi(t) D(x t)$. Now $0 \leqq D(x t) \leqq 1$, and the hypothesis that $\phi(t)$ is non-negative and monotone decreasing over $t>0$ and $\int_{0}^{\infty} \phi(t) d t=1$ implies that $t \phi(t) \rightarrow 0$ as $t \rightarrow \infty$ and $t \phi(t) \rightarrow 0$ as $t \rightarrow 0$. Hence $\phi(t) S(x t) \rightarrow 0$ as $t \rightarrow \infty$ and $\phi(t) S(x t) \rightarrow 0$ as $t \rightarrow 0$. Therefore integrating (3.1) by parts gives

$$
F(x)=-\int_{0}^{\infty} D(x t) t d \phi(t) .
$$

Defining lower densities by the formulas $L D=\lim _{\inf _{x \rightarrow \infty}} D(x)$ and $L D_{\phi}$ $=\lim _{\inf _{x \rightarrow \infty}} F(x)$, we can use (3.12) to prove that 


$$
L D \leqq L D_{\phi} \leqq \bar{D}_{\phi} \leqq \bar{D} .
$$

Proof of the first of the inequalities (3.2) being similar, we prove only the last. Let $\epsilon>0$ and choose a positive number $\delta$ such that

$$
\int_{0}^{\delta} \phi(t) d t<\epsilon
$$

Choose $x_{0}$ such that $D(x)<\bar{D}+\epsilon$ when $x>x_{0}$. Then, when $x>x_{0} / \delta$,

$$
F(x) \leqq-\int_{0}^{\delta} t d \phi(t)-(\bar{D}+\epsilon) \int_{\delta}^{\infty} t d \phi(t) .
$$

Integration by parts gives

$$
-\int_{0}^{\delta} t d \phi(t)=-\delta \phi(\delta)+\int_{0}^{\delta} \phi(t) d t<\epsilon
$$

and

$$
-\int_{\delta}^{\infty} t d \phi(t)<-\int_{0}^{\infty} t d \phi(t)=\int_{0}^{\infty} \phi(t) d t=1 .
$$

Hence, when $x>x_{0}, F(x)<\bar{D}+2 \epsilon$ and this implies that $\bar{D}_{\phi} \leqq \bar{D}$.

That the following theorem gives much more incisive information about $\bar{D}_{\phi}$ is shown by applying (3.31) in the special case for which $\bar{D}=1$; putting $\bar{D}=1$ in (3.31) gives immediately the significant conclusion that $\bar{D}_{\phi}=1$. It follows from this, (3.2), and the inequality $0 \leqq \bar{D} \leqq 1$ that if $\bar{D}_{\phi}=1$ for at least one $\phi$, then $\bar{D}_{\phi}=1$ for every $\phi$. Another obvious consequence of (3.31) and (3.311) is the fact that if $\bar{D}>0$, then $\bar{D}_{\phi}>0$ for every $\phi$.

TheOREM 3.3. For each set $E$ of positive integers, the upper density $\bar{D}_{\phi}$ and the upper density $\bar{D}$ satisfy the condition

$$
\underset{x>0}{\text { l.u.b. }} \int_{(1-\bar{D}) x}^{x} \phi(t) d t \leqq \bar{D}_{\phi} \leqq \bar{D}
$$

and hence also the weaker but simpler condition

$$
\left[\max _{t>0} t \phi(t)\right] \bar{D} \leqq \bar{D}_{\phi} \leqq \bar{D} .
$$

Moreover to each number $\theta$ for which $0 \leqq \theta \leqq 1$ there correspond sets $E$ of positive integers for which $\bar{D}=\theta$ and

$$
\bar{D}_{\phi}=\underset{x>0}{\text { l.u.b. }} \int_{(1-\theta) x}^{x} \phi(t) d t .
$$

The last inequality in (3.31) follows from (3.2). The part of Theorem 3.3 
involving (3.32) will be proved in $\S \S 4$ and 5 where considerable further information will be obtained. Meanwhile in this section we give a proof of the first inequality in (3.31) and obtain (3.311) and related results. Let $x>0$. Then, for each $y>0$, starting with (3.1) gives

$$
F(y) \geqq \frac{1}{y} \int_{0}^{x} \phi(t) d S(y t)=\frac{1}{y} \int_{0}^{x y} \phi\left(\frac{u}{y}\right) d S(u) .
$$

Since $S(u)$ is constant over $0 \leqq u \leqq x y$ except for $S(x y)$ upward jumps of unit magnitude and $\phi$ is monotone decreasing, it follows that

$$
F(y) \geqq \frac{1}{y} \int_{x y-S(x y)}^{x y} \phi\left(\frac{u}{y}\right) d u=\int_{x-x[S(x y) / x y]}^{x} \phi(t) d t .
$$

Letting $y$ become infinite over a sequence of values for which $S(x y) / x y \rightarrow \bar{D}$ then gives the first inequality in (3.31).

We now show that (3.11) implies (3.311). As we have remarked, our hypotheses imply that $t \phi(t) \rightarrow 0$ as $t \rightarrow 0$ and as $t \rightarrow \infty$. Since $\phi(t)$ is continuous, non-negative, and sometimes positive over $t>0$, it follows that $t \phi(t)$ must attain a positive absolute maximum $C$ for some positive value $t_{0}$ of $t$. Since $\phi(t)$ is monotone decreasing over $t>0$, we find that

$$
\begin{aligned}
& \underset{x>0}{\text { l.u.b. }} \int_{(1-\bar{D}) x}^{x} \phi(t) d t \geqq \text { l.u.b. } \int_{x>0}^{x} \phi(x) d t \\
& =\underset{x>0}{\text { l.u.b. }} \bar{D} x \phi(x)=\left[\max _{x>0} x \phi(x)\right] \bar{D} \text {. }
\end{aligned}
$$

This and (3.31) imply (3.311).

Further information about the left member of (3.31) is easily obtained. Using again the fact that $\phi(t)$ is monotone decreasing gives, when $0 \leqq \bar{D}<1$,

$$
\begin{aligned}
\underset{x>0}{\text { l.u.b. }} \int_{(1-\bar{D}) x}^{x} \phi(t) d t \leqq \underset{x>0}{\text { l.u.b. }} \int_{(1-\bar{D}) x}^{x} \phi((1-\bar{D}) x) d t \\
\quad=\frac{1}{1-D} \text { l.u.b. }[(1-\bar{D}) x \phi((1-\bar{D}) x)] \bar{D}=\frac{1}{1-\bar{D}}\left[\max _{x>0} x \phi(x)\right] \bar{D} .
\end{aligned}
$$

When $0<D<1,(3.6)$ and (3.61) give

$$
\max _{x>0} x \phi(x) \leqq \operatorname{liu}_{x>0} . \mathrm{b} . \quad \frac{1}{\bar{D}} \int_{(1-\bar{D}) x}^{x} \phi(t) d t \leqq \frac{1}{1-\bar{D}} \max _{x>0} x \phi(x) .
$$

This implies that

$$
\lim _{\bar{D} \rightarrow 0} \underset{x>0}{\text { l.u.b. }} \frac{1}{\bar{D}} \int_{(1-\bar{D}) x}^{x} \phi(t) d t=\max _{x>0} x \phi(x) .
$$


Since $\phi(t) \geqq 0$ and, for each fixed $x$, an increase in $\bar{D}$ produces an enlargement of the interval of integration in the integral in the left member of (3.61), it follows that the left member of (3.61) is a monotone increasing function of $\bar{D}$. Some of our later work suggests that, for the special cases in which we are interested, the left member of (3.61) is a function of $\bar{D}$ having positive derivatives of all orders, and that the central member of (3.62) is an increasing function of $\bar{D}$.

4. Development of a method. We now develop a method for constructing and using special sequences $s_{n}$ for the purpose of obtaining information about the manner in which $\bar{D}_{M}^{(r)}, \bar{D}_{A}, \bar{D}_{a}$ and $\bar{D}_{P}$ are related to $\bar{D}$ and to each other. One of our goals is to complete the proof of Theorem 3.3 by proving the statement involving (3.32). A part of this method has been suggested in a special case by Dvoretzky [1] who gives references to earlier work of $S$. Mandelbrojt and others. As in $\S 3$, we assume that $\phi(t) \geqq 0, \phi(t)$ is continuous and monotone decreasing over $t>0$, and $\int_{0}^{\infty} \phi(t) d t=1$. Each set $E$ of positive integers $\lambda_{1}, \lambda_{2}, \cdots$ then possesses, as in $\S 3$, a transform $F(x)$ defined by

$$
F(x)=\frac{1}{x} \int_{0}^{\infty} \phi(t) d S(x t)
$$

and an upper density $D_{\phi}$ defined by

$$
\bar{D}_{\phi}=\limsup _{x \rightarrow \infty} F(x) .
$$

For application to special cases we construct, in terms of the function $\phi(t)$ in (4.1) and a given constant $\theta$ for which $0 \leqq \theta \leqq 1$, a special sequence of zeros and ones which we denote by $s_{n}$. Let $a_{1}, b_{1}, a_{2}, b_{2}, \cdots$ be an increasing sequence of positive integers and let $x_{1}, x_{2}, \cdots$ be an increasing sequence of positive numbers satisfying the conditions $x_{n} \rightarrow \infty$,

$$
\begin{aligned}
\lim _{p \rightarrow \infty}\left(b_{p}-a_{p}\right) & =\infty, & \lim _{p \rightarrow \infty}\left(a_{p+1}-b_{p}\right) & =\infty, \\
\lim _{p \rightarrow \infty} \frac{b_{p}}{a_{p+1}} & =0, & \lim _{p \rightarrow \infty} \frac{b_{p}-a_{p}}{b_{p}} & =\theta, \\
\int_{0}^{b_{p} / x_{p}} \phi(t) d t & <\frac{1}{p}, & \int_{a_{p+1} / x_{p}}^{\infty} \phi(t) d t & <\frac{1}{p} .
\end{aligned}
$$

The order of choice of these numbers can be $a_{1}, b_{1}, x_{1}, a_{2}, b_{2}, x_{2}, \cdots$. Assuming that $a_{1}$ and $b_{1}$ are given such that $a_{1}<b_{1}$, we can choose $x_{1}>0$ and then choose $a_{2}$ as large as we please to validate (4.22) when $p=1$. It is then easy to choose $b_{2}$ and to continue the process to validate (4.2), (4.21), and (4.22). Let $s_{n}=1$ when $n$ falls within one of the intervals $a_{p}<n \leqq b_{p}$ and let $s_{n}=0$ when $n \leqq a_{1}$ and when $n$ falls within one of the intervals $b_{p}<n \leqq a_{p+1}$. Thus the sequence $s_{n}$ consists of alternate blocks of zeros and ones, the lengths of 
these blocks becoming infinite as we advance in the sequence. Using (4.21) and the definition (1.2) of $D(t)$, we find that

$$
\lim _{p \rightarrow \infty} D\left(a_{p}\right)=0, \quad \lim _{p \rightarrow \infty} D\left(b_{p}\right)=\theta
$$

and that

$$
\bar{D}=\limsup _{t \rightarrow \infty} D(t)=\theta .
$$

For each $p=1,2, \cdots$, let $s_{1}^{(p)}, s_{2}^{(p)}, \cdots$ denote the sequence for which $s_{n}^{(p)}=0$ when $n \leqq a_{p}, s_{n}^{(p)}=1$ when $a_{p}<n \leqq b_{p}$, and $s_{n}^{(p)}=0$ when $n>b_{p}$. Thus the sequence $s_{1}^{(p)}, s_{2}^{(p)}, \ldots$ consists of an initial block of zeros, a single block of ones, and a terminal infinite block of zeros. The sequence $s_{n}$ is the elementwise sum of all of the sequences $s_{n}^{(1)}, s_{n}^{(2)}, \cdots$. For each $p=1,2, \cdots$, let $S_{p}(t)$ denote the counting function of the sequence $s_{n}^{(p)}$. The transforms by (4.1) of the sequences $s_{n}^{(p)}$ and $s_{n}$ are then respectively

$$
F_{p}(x)=\frac{1}{x} \int_{0}^{\infty} \phi(t) d S_{p}(x t)
$$

and

$$
F(x)=\sum_{k=1}^{\infty} F_{k}(x)
$$

The function $S_{p}(x t)$ is constant over $t \geqq 0$ except for unit upward jumps at the points for which $x t$ is $a_{p}+1, a_{p}+2, \cdots, b$ and hence at the points for which $t$ is $\left(a_{p}+1\right) / t,\left(a_{p}+2\right) / t, \cdots, b / t$. Hence (4.33) implies that

$$
F_{p}(x)=\sum_{n=a_{p}+1}^{b_{p}} \phi\left(\frac{n}{x}\right) \frac{1}{x} .
$$

The right member of (4.35) is a simple Riemann sum formed for the function $\phi(t)$ over the interval $\left(\left(a_{p}+1\right) / x, b_{p} / x\right)$ and, because $\phi(t)$ is monotone decreasing, this sum is less than or equal to the corresponding integral. Therefore (4.35) implies that

$$
F_{p}(x) \leqq \int_{a_{p} / x}^{b_{p} / x} \phi(t) d t
$$

Hence when $p>1$

$$
\sum_{k=1}^{p-1} F_{k}(x) \leqq \int_{0}^{b_{p-1} / x} \phi(t) d t, \quad \sum_{k=p+1}^{\infty} F_{k}(x) \leqq \int_{a_{p+1} / x}^{\infty} \phi(t) d t .
$$

In case $x_{p-1} \leqq x \leqq x_{p}$, this and (4.22) imply that 


$$
\sum_{k=1}^{p-1} F_{k}(x)=\epsilon_{p}, \quad \sum_{k=p+1}^{\infty} F_{k}(x)=\epsilon_{p}
$$

where $\epsilon_{p}$ stands generically for a non-negative quantity less than $1 /(p-1)$. Starting with (4.11) and using (4.34) and (4.38) gives

$$
\begin{aligned}
\bar{D}_{\phi} & =\limsup _{p \rightarrow \infty} \underset{\substack{\text { l.u.b. } \\
x_{p-1} \leqq x \leqq x_{p}}}{ } F(x) \\
& =\limsup _{p \rightarrow \infty} \underset{\substack{\text { l.u.b. } \\
x_{p-1} \leqq x \leqq x_{p}}}{ }\left[2 \epsilon_{p}+F_{p}(x)\right] \\
& =\limsup _{p \rightarrow \infty} \underset{\substack{\text { l.u.b. } \\
x_{p-1} \leqq x \leqq x_{p}}}{ } F_{p}(x) .
\end{aligned}
$$

We now use again the representation of $F_{p}(x)$ as the Riemann sum in (4.35). When $p \rightarrow \infty$ and $x_{p-1} \leqq x \leqq x_{p}$, so that $1 / x \rightarrow 0$, our hypotheses on $\phi(t)$ imply that the Riemann sum differs from the corresponding integral by $o(1)$. Therefore (4.4) implies that

$$
\bar{D}_{\phi}=\limsup _{p \rightarrow \infty} \underset{x_{p-1} \leqq x \leqq x_{p}}{\text { l.u.b. }} \int_{a_{p} / x}^{b_{p} / x} \phi(t) d t .
$$

When $x \leqq x_{p-1}$ and when $x \geqq x_{p}$, (4.22) shows that

$$
\int_{a_{p} / x}^{b_{p} / x} \phi(t) d t=2 \epsilon_{p}
$$

Hence (4.41) implies that

$$
\bar{D}_{\phi}=\limsup _{p \rightarrow \infty} \text { 1.u.b. } \int_{x>0}^{b_{p} / x} \phi(t) d t .
$$

Using the second of the relations (4.2), we define $\theta_{p}$ by the formula

$$
\theta_{p}=\left(b_{p}-a_{p}\right) / b_{p}
$$

and observe that $\theta_{p} \rightarrow \theta$ as $p \rightarrow \infty$. Using (4.44) puts (4.43) in the form

$$
\bar{D}_{\phi}=\limsup _{p \rightarrow \infty} \operatorname{liu}_{x>0} \int_{\left(1-\theta_{p}\right) b_{p} / x}^{b_{p} / x} \phi(t) d t
$$

and hence

$$
\bar{D}_{\phi}=\underset{p \rightarrow \infty}{\limsup } \text { l.u.b. } \int_{x>0}^{x} \phi(t) d t .
$$

Two applications of our results come from consideration of the extreme cases in which $\theta=0$ and $\theta=1$. In case $\theta=0$ so that $\bar{D}=0$ and $\theta_{p} \rightarrow 0,(4.46)$ implies that $\bar{D}_{\phi}=0$. In case $\theta=1$ so that $\bar{D}=1$ and $\theta_{p} \rightarrow 1,(4.46)$ implies that 


$$
\bar{D}_{\phi}=\int_{0}^{\infty} \phi(t) d t=1 .
$$

Henceforth we confine our attention to the case for which $0<\theta<1$. In this case, (4.46) implies that

$$
\bar{D}_{\phi}=\underset{x>0}{\text { l.u.b. }} \int_{(1-\theta) x}^{x} \phi(t) d t .
$$

The integral in (4.5) is a continuous non-negative function of $x$ which converges to 0 as $x \rightarrow 0$ and as $x \rightarrow \infty$. This function must attain a maximum value for at least one $x$ for which $x>0$ and hence (4.5) takes the form

$$
\bar{D}_{\phi}=\max _{x>0} \int_{(1-\theta) x}^{x} \phi(t) d t .
$$

5. Continuation of the method. For convenience of reference, we summarize, in the following theorem, the results from $\$ 4$ that we want to use. This theorem implies the last part of Theorem 3.3 and hence the proof of Theorem 3.3 is now complete.

THEOREM 5.1. Let $\phi(t)$ be non-negative, continuous, and monotone decreasing over $t>0$, and let

$$
\int_{0}^{\infty} \phi(t) d t=1
$$

Let the upper density $\bar{D}_{\phi}$ of a sequence $s_{n}$ of zeros and ones be defined by

$$
\bar{D}_{\phi}=\limsup _{x \rightarrow \infty} \frac{1}{x} \int_{0}^{\infty} \phi(t) d S(x t) .
$$

Let $0<\theta<1$ and let $\lambda=1-\theta$. Let $s_{n}$ be the special sequence of zeros and ones constructed in $\S 4$. Then $\bar{D}=\theta$ and

$$
\bar{D}_{\phi}=\max _{x>0} \int_{\lambda x}^{x} \phi(t) d t .
$$

If $\theta=0$, then $\bar{D}_{\phi}=0$ and if $\theta=1$, then $\bar{D}_{\phi}=1$.

We suppose henceforth that $\theta$ is fixed such that $0<\theta<1$ and simplify formulas by putting $\lambda=1-\theta$. Let

$$
f(x)=\int_{\lambda x}^{x} \phi(t) d t
$$

so that, by (5.13), $\bar{D}_{\phi}=\max f(x)$. The function $f(x)$ is differentiable, nonnegative, and sometimes positive over $x>0$, and $f(x) \rightarrow 0$ as $x \rightarrow 0$ and as $x \rightarrow \infty$. 
Hence $f(x)$ attains an absolute positive maximum for some positive value of $x$ for which $f^{\prime}(x)=0$. The positive zeros of $f^{\prime}(x)$ are the same as those of the function $g(x)$ for which $g(x)=x f^{\prime}(x)$ and hence

$$
g(x)=x \phi(x)-\lambda x \phi(\lambda x) .
$$

In cases in which it is not easy to solve the equation $f^{\prime}(x)=0$, a graph of $x \phi(x)$ gives approximations to pairs of values of $x$ and $\lambda$ for which $g(v)=0$.

TABLE 1

\begin{tabular}{|c|c|c|c|c|c|c|c|c|}
\hline$\theta=\bar{D}$ & \multicolumn{2}{|l|}{$\bar{D}_{M}^{(1)}$} & \multicolumn{2}{|l|}{$\bar{D}_{\boldsymbol{\mu}}^{(2)}$} & \multicolumn{2}{|c|}{$\bar{D}_{\boldsymbol{M}}^{(3)}$} & \multicolumn{2}{|c|}{$\bar{D}_{\mu}^{(4)}$} \\
\hline 0 & 0 & & 0 & & 0 & & 0 & \\
\hline .1 & .03874 & 20 & .02851 & 14 & .02360 & 15 & .02058 & 16 \\
\hline .2 & .08192 & 00 & .06033 & 58 & .04995 & 89 & .04357 & 22 \\
\hline .3 & .13052 & 19 & .09628 & 62 & .07976 & 91 & .06959 & 02 \\
\hline .4 & .18590 & 32 & .13751 & 79 & .11403 & 30 & .09952 & 78 \\
\hline .5 & .25000 & 00 & .18575 & 53 & .15426 & 43 & .13474 & 34 \\
\hline .6 & .32573 & 01 & .24374 & 96 & .20292 & 03 & .17746 & 01 \\
\hline .7 & .41783 & 72 & .31632 & 83 & .26441 & 37 & .23171 & 50 \\
\hline .8 & .53499 & 22 & .41333 & 02 & .34804 & 72 & .30615 & 96 \\
\hline .85 & .60816 & 76 & .47770 & 03 & .40477 & 39 & .35721 & 96 \\
\hline .9 & .69683 & 73 & .56132 & 81 & .48043 & 61 & .42625 & 29 \\
\hline .95 & .81142 & 49 & .68355 & 45 & .59664 & 46 & .53507 & 38 \\
\hline .99 & .94500 & 30 & .86956 & 21 & .79714 & 66 & .73630 & 67 \\
\hline .999 & .99211 & 61 & .97158 & 80 & .93943 & 34 & .90287 & 86 \\
\hline 1 & 1 & & 1 & & 1 & & 1 & \\
\hline
\end{tabular}

TABLE 2

\begin{tabular}{|c|c|c|c|c|c|c|c|c|}
\hline$\theta=\bar{D}$ & \multicolumn{2}{|c|}{$\bar{D}_{M}=\bar{D}_{a}$} & \multicolumn{2}{|c|}{$\bar{D}_{A}$} & \multicolumn{2}{|l|}{$\bar{D}_{P}$} & \multicolumn{2}{|c|}{$\bar{D}_{\boldsymbol{\mu}}^{(5)}$} \\
\hline 0 & 0 & & 0 & & 0 & & 0 & \\
\hline .1 & .03874 & 20 & .02965 & 06 & .02698 & 15 & .01848 & 56 \\
\hline .2 & .08192 & 00 & .06273 & 99 & .05708 & 96 & .03913 & 82 \\
\hline .3 & .13052 & 19 & .10010 & 19 & .09107 & 87 & .06251 & 85 \\
\hline .4 & .18590 & 32 & .14291 & 63 & .13001 & 49 & .08943 & 87 \\
\hline .5 & .25000 & 00 & 19293 & 53 & .17547 & 97 & .12113 & 96 \\
\hline .6 & .32573 & 01 & .25293 & 60 & .22997 & 92 & .15966 & 16 \\
\hline .7 & .41783 & 72 & .32774 & 98 & .29787 & 98 & .20873 & 47 \\
\hline .8 & .53499 & 22 & .42710 & 87 & .38802 & 72 & .27642 & 96 \\
\hline .85 & .60816 & 76 & .49254 & 34 & .44746 & 90 & .32317 & 38 \\
\hline .9 & .69683 & 73 & .57682 & 73 & .52433 & 80 & .38689 & 42 \\
\hline .95 & .81142 & 49 & .69821 & 97 & .63659 & 52 & .48895 & 17 \\
\hline .99 & .94500 & 30 & .87774 & 82 & .81388 & 07 & .68605 & 62 \\
\hline .999 & .99211 & 61 & .97315 & 87 & .93258 & 94 & .86639 & 31 \\
\hline 1 & 1 & & 1 & & 1 & & 1 & \\
\hline
\end{tabular}


Newton's method then provides an efficient means of approximating zeros of $g(x)$ and hence of $f^{\prime}(x)$.

6. The tables. Before discussing the accompanying tables, we remark that the entries in Tables 1 and 3 were obtained by the methods set forth in $\S \S 4$ and 5. Some details and further information are given in $\$ \S 7$ to 10 .

Table 1 gives, for each of several values of $\theta$, the values of $\bar{D}, \bar{D}_{M}^{(1)}, \bar{D}_{M}^{(2)}$, $\bar{D}_{M}^{(3)}$ and $\bar{D}_{M}^{(4)}$ for the special sequences of zeros and ones constructed in $\S 4$. Table 2 gives the corresponding values of $\bar{D}_{M}, \bar{D}_{a}, \bar{D}_{A}, \bar{D}_{P}$ and $\bar{D}_{M}^{(5)}$. Table 3 gives, for the same values of $\theta$, the values of the differences $\bar{D}-D_{M}^{(1)}, \bar{D}_{M}^{(1)}$ $-\bar{D}_{M}^{(2)}, \bar{D}_{M}^{(2)}-\bar{D}_{M}^{(3)}, \bar{D}_{M}^{(3)}-\bar{D}_{M}^{(4)}$ (obtained from Table 1) and $\bar{D}_{M}^{(4)}-\bar{D}_{M}^{(5)}$ (obtained from Tables 1 and 2). The most interesting fact revealed by Table 3 is the fact that the sequence

$$
1-\bar{D}, \bar{D}-\bar{D}_{M}^{(1)}, \bar{D}_{M}^{(1)}-\bar{D}_{M}^{(2)}, \bar{D}_{M}^{(2)}-\bar{D}_{M}^{(3)}, \bar{D}_{M}^{(3)}-\bar{D}_{M}^{(4)}, \cdots
$$

is not monotone when $\bar{D}$ has the values $.8, .85, .9, .95, .99$, and .999 . Table 4 gives, in decreasing order through the first column and then the second column, the values of several upper densities when $\theta=.999$. These upper densities maintain the same relative sizes for all of the values of $\theta$ listed in Tables 1 and 2. Following the numbers in Table 4, we give the interpretations of these numbers in terms of transforms of the sequence $s_{n}$; the notation is defined at the end of $\$ 1$.

We conclude this section with some remarks concerning the relative sizes of the numbers in Table 4 . At widely spaced times, the author has participated in informal discussions of the following idea. In comparison to the

TABLE 3

\begin{tabular}{c|c|c|c|c|c|c}
\hline \hline$\theta=\bar{D}$ & $1-\bar{D}$ & $\bar{D}-\bar{D}_{\boldsymbol{\mu}}^{(1)}$ & $\bar{D}_{\boldsymbol{\mu}}^{(1)}-\bar{D}_{\boldsymbol{\mu}}^{(2)}$ & $\bar{D}_{\boldsymbol{\mu}}^{(2)}-\bar{D}_{\boldsymbol{\mu}}^{(3)}$ & $\bar{D}_{\boldsymbol{\mu}}^{(3)}-\bar{D}_{\boldsymbol{\mu}}^{(4)}$ & $\bar{D}_{\boldsymbol{\mu}}^{(4)}-\bar{D}_{\boldsymbol{\mu}}^{(5)}$ \\
\hline & 1 & 0 & 0 & 0 & 0 & 0 \\
\hline 0 & .9 & .06126 & .01023 & .00491 & .00302 & .00210 \\
.1 & .8 & .11808 & .02158 & .01038 & .00639 & .00443 \\
.2 & .7 & .16948 & .03424 & .01652 & .01018 & .00707 \\
.3 & .6 & .21410 & .04839 & .02348 & .01451 & .01009 \\
.4 & .6 & .25000 & .06424 & .03149 & .01952 & .01360 \\
.5 & .5 & .27427 & .08198 & .04083 & .02546 & .01780 \\
.6 & .4 & .28216 & .10151 & .05191 & .03270 & .02298 \\
.7 & .3 & .26501 & .12166 & .06528 & .04189 & .02973 \\
.8 & .2 & .24183 & .13047 & .07293 & .04755 & .03405 \\
.85 & .15 & .20316 & .13551 & .08089 & .05418 & .03936 \\
.9 & .1 & .13858 & .12787 & .08691 & .06157 & .04612 \\
.95 & .05 & .04500 & .07544 & .07242 & .06084 & .05025 \\
.99 & .01 & .00688 & .02053 & .03215 & .03655 & .03649 \\
.999 & .001 & .0035 & & 0 & 0 \\
1 & 0 & 0 & 0 & 0 & 0 & \\
\hline
\end{tabular}


TABLE 4

\begin{tabular}{|c|c|c|c|}
\hline $\bar{S}=1.00000$ & $00=\bar{S}$ & $\bar{D}_{\boldsymbol{M}}^{(2)}=.97158$ & $80=\bar{M}^{(3)}$ \\
\hline $\bar{D}=.99900$ & $00=\bar{M}^{(1)}$ & $\bar{D}^{(3)}=.93943$ & $34=\bar{M}^{(4)}$ \\
\hline $\bar{D}_{\boldsymbol{\mu}}^{(1)}=.99211$ & $61=\bar{M}^{(2)}$ & $\bar{D}_{P}=.93258$ & $94=\bar{P} \bar{M}$ \\
\hline $\bar{D}_{a}=.99211$ & $61=\bar{A}$ & $\bar{D}_{\boldsymbol{M}}^{(4)}=.90287$ & $86=\bar{M}^{(5)}$ \\
\hline $\bar{D}_{A}=.97315$ & $87=\bar{A} \bar{M}$ & $\bar{D}_{\mathcal{M}}^{(5)}=.86639$ & $31=\bar{M}^{(6)}$ \\
\hline
\end{tabular}

arithmetic mean transformation $M$, the Abel transformation $A$ is an averaging process so strong that the $A$ and $A M$ transforms of each bounded sequence should be asymptotically equal and hence we should have $\bar{A}=\bar{A} \bar{M}$. Arguments in favor of validity of this idea receive a little support from a theorem of Zygmund which has been generalized by Szász [2] and which says that if a sequence is evaluable $A$, then it is evaluable $A M$. However, the idea is false because Table 4 shows that there exist sequences of zeros and ones for which $\bar{A} \bar{M}<\bar{A}$. The above idea which has already been discredited and known relations involving the Abel and Cesàro transforms of series, together with the fact that the Hölder and Cesàro methods for evaluation of sequences are equivalent, could lead to the guess that if $r>0$ then the inequality $\bar{A} \leqq \bar{M}^{(r)}$ holds for each real sequence. However, Table 3 shows that there exist sequences of zeros and ones for which $M^{(3)}<\bar{A}$.

7. The density $\bar{D}_{M}^{(r)}$. In accordance with Theorem 5.1 and (2.21), $\bar{D}_{M}^{(r)}$ $=\max _{x>0} f(x)$ where

$$
f(x)=\frac{1}{r !} \int_{\lambda x}^{x}\left(\log ^{+} \frac{1}{t}\right)^{r} d t
$$

and $\log ^{+} u$ is 0 or $\log u$ according as $0<u<1$ or $u \geqq 1$. The maximum value of $f(x)$ occurs for the unique $x$ for which $f^{\prime}(x)=0$ and hence

$$
\log \frac{1}{x}=\frac{\lambda^{1 / r}}{1-\lambda^{1 / r}} \log \frac{1}{\lambda} .
$$

To determine $\bar{D}_{M}^{(r)}$, we integrate (7.1) by parts to obtain

$$
\bar{D}_{M}^{(r)}=x \sum_{k=0}^{r-1} \frac{1}{k !}\left[1-\lambda^{(r-k) / r}\right]\left(\log \frac{1}{x}\right)^{k}
$$

where $x$ is determined from (7.2). In case $r=1$, use of (7.3) and (7.2) shows that

$$
\bar{D}_{M}=\bar{D}_{M}^{(1)}=(1-\lambda) x=(1-\lambda) \lambda^{\lambda /(1-\lambda)}
$$


and hence, since $\lambda=1-\theta$, that

$$
\bar{D}_{M}=\bar{D}_{M}^{(1)}=\theta(1-\theta)^{(1-\theta) / \theta} .
$$

An illuminating formula for $\bar{D}_{M}^{(r)}$ arises from making a change of the variable of integration in (7.1). Putting $t=e^{-u}$ gives

$$
\bar{D}_{M}^{(r)}=\max _{0<x<1} \frac{1}{r !} \int_{\log x^{-1}}^{\log x^{-1}+\log \lambda^{-1}} u^{r} e^{-u} d u
$$

and a change of notation gives

$$
\bar{D}_{M}^{(r)}=\max _{y>0} \frac{1}{r !} \int_{y}^{y+\log \lambda^{-1}} t^{r} e^{-t} d t .
$$

This and elementary properties of the function $t^{r} e^{-t}$, including the fact that $0 \leqq t^{r} e^{-t} \leqq r^{r} e^{-r}$ when $t \geqq 0$, imply that

$$
\bar{D}_{M}^{(r)} \leqq \frac{r^{r} e^{-r}}{r !} \log \frac{1}{\lambda}
$$

and that, for each fixed $r$,

$$
\lim _{\lambda \rightarrow 1} \bar{D}_{M}^{(r)} /\left(\log \frac{1}{\lambda}\right)=\frac{r^{r} e^{-r}}{r !} .
$$

From (7.62) and Stirling's formula for $r$ !, we see that

$$
\bar{D}_{M}^{(r)} \leqq \frac{1}{(2 r \pi)^{1 / 2}} \log \frac{1}{\lambda}
$$

and hence that, for each fixed $\lambda, \bar{D}_{M}^{(r)} \rightarrow 0$ as $r \rightarrow \infty$. In terms of the variable $\theta$, (7.63) gives

$$
\lim _{\theta \rightarrow 0} \bar{D}_{M}^{(r)} / \theta=\frac{r^{r} e^{-r}}{r !} .
$$

Some numerical values are

$$
\begin{aligned}
& \lim _{\theta \rightarrow 0} \bar{D}_{M}^{(1)} / \theta=.3678794412, \\
& \lim _{\theta \rightarrow 0} \bar{D}_{M}^{(2)} / \theta=.2706705665, \\
& \lim _{\theta \rightarrow 0} \bar{D}_{M}^{(3)} / \theta=.2240418077, \\
& \lim _{\theta \rightarrow 0} \bar{D}_{M}^{(4)} / \theta=.1953668148, \\
& \lim _{\theta \rightarrow 0} \bar{D}_{M}^{(5)} / \theta=.1754673698 .
\end{aligned}
$$


8. The density $\bar{D}_{a}$. In accordance with Theorem 5.1 and (2.23), $\bar{D}_{a}$ $=\max _{x>0} f(x)$ where

$$
f(x)=\int_{\lambda x}^{x} e^{-t} d t
$$

The maximum value of $f(x)$ occurs for the unique value of $x$ for which $f^{\prime}(x)$ $=0$, that is

$$
e^{-x}-\lambda e^{-\lambda x}=0
$$

or

$$
x=(\log \lambda) /(\lambda-1) .
$$

When $x$ has the value in (8.2) and (8.3), we find by evaluating the integral in (8.1) that

$$
\bar{D}_{a}=f(x)=e^{-\lambda x}-e^{-x}=(1-\lambda) e^{-\lambda x}=(1-\lambda) \lambda^{\lambda /(1-\lambda)}
$$

and, since $\lambda=1-\theta$, that

$$
\bar{D}_{a}=\theta(1-\theta)^{(1-\theta) / \theta} \text {. }
$$

Thus $\bar{D}_{a}=\bar{D}_{M}$.

The same change of variable of integration that led from (7.1) to (7.6) leads from (8.1) to the formula

$$
\bar{D}_{a}=\max _{-\infty<y<\infty} \int_{y}^{y+\log \lambda^{-1}} e^{-t} e^{-e^{-t}} d t .
$$

9. The density $\bar{D}_{A}$. In accordance with Theorem 5.1 and (2.22), $\bar{D}_{A}$ $=\max _{x>0} f(x)$ where

$$
f(x)=\int_{\lambda x}^{x} E(t) d t .
$$

The maximum value of $f(x)$ occurs for the unique $x$ for which $f^{\prime}(x)=0$, that is

$$
E(x)-\lambda E(\lambda x)=0 .
$$

When $\lambda$ has a given value, the main difficulty in applying Newton's method to solve (9.2) for $x$ lies in obtaining sufficiently accurate values of $E(y)$. When $E(a)$ is tabulated, the value of $E(a+h)$ can be obtained correct to the desired number of decimal places by use of the Taylor expansion of $E(a+h)$ in powers of $h$, which can be put in the convenient form

$$
E(a+h)=E(a)-R e^{-a}
$$

where 


$$
\begin{aligned}
R= & \frac{h}{a}-\frac{1+a}{2}\left(\frac{h}{a}\right)^{2}+\frac{2+2 a+a^{2}}{6}\left(\frac{h}{a}\right)^{3} \\
& -\frac{6+6 a+3 a^{2}+a^{3}}{24}\left(\frac{h}{a}\right)^{4}+\cdots .
\end{aligned}
$$

When $x$ has been determined from (9.2), evaluation of $\bar{D}_{A}$ is easy. In fact, for each $y>0$,

$$
\begin{aligned}
\int_{y}^{\infty} E(t) d t & =\int_{y}^{\infty} d t \int_{t}^{\infty} \frac{e^{-u}}{u} d u \\
& =\int_{y}^{\infty} d u \int_{y}^{u} \frac{e^{-u}}{u} d t=\int_{y}^{\infty} \frac{u-y}{u} e^{-u} d u=e^{-y}-y E(y) .
\end{aligned}
$$

Hence, when $x$ has the value determined by (9.2), use of (9.1) and (9.3) gives

$$
\bar{D}_{A}=f(x)=\left[e^{-\lambda x}-\lambda x E(\lambda x)\right]-\left[e^{-x}-x E(x)\right]
$$

and hence

$$
\bar{D}_{A}=e^{-\lambda x}-e^{-x} \text {. }
$$

Changing the variable of integration in (9.1) by putting $t=e^{-u}$ gives

$$
\bar{D}_{A}=\max _{x>0} \int_{\log x^{-1}}^{\log x^{-1}+\log \lambda^{-1}} E\left(e^{-u}\right) e^{-u} d u
$$

and hence

$$
\bar{D}_{A}=\max _{-\infty<y<\infty} \int_{y}^{y+\log \lambda^{-1}} E\left(e^{-t}\right) e^{-t} d t .
$$

The function $x E(x)$ attains its maximum value when $x=.43481820$ and the maximum value is the constant $C_{A}$ having the value

$$
C_{A}=.281493630 \text {. }
$$

Hence (8.7) implies that

$$
\bar{D}_{A} \leqq C_{A} \log \lambda^{-1}
$$

and that

$$
\lim _{\lambda \rightarrow 1} \bar{D}_{A} / \log \lambda^{-1}=C_{A}
$$

In terms of $\theta,(9.73)$ gives

$$
\lim _{\theta \rightarrow 0} \bar{D}_{A} / \theta=C_{A}
$$


10. The density $\bar{D}_{P}$. In accordance with Theorem 5.1 and (2.24), $\bar{D}_{P}$ $=\max _{x>0} f(x)$ where

$$
f(x)=\frac{1}{\pi} \int_{\lambda x}^{x} \log \left(1+\frac{1}{t^{2}}\right) d t .
$$

The maximum value of $f(x)$ occurs for the unique value of $x$ for which $f^{\prime}(x)$ $=0$, that is

$$
\log \left(1+\frac{1}{x^{2}}\right)-\lambda \log \left(1+\frac{1}{\lambda^{2} x^{2}}\right)=0 .
$$

As in previous cases, when $x$ has been determined from (10.2), evaluation of $\bar{D}_{P}$ is easier than might be expected. When $x$ has the value determined from (10.2), evaluation of the integral in (10.1) gives

$$
\bar{D}_{P}=\frac{1}{\pi}\left[x \log \left(1+\frac{1}{x^{2}}\right)-\lambda x \log \left(1+\frac{1}{\lambda^{2} x^{2}}\right)\right.
$$

$$
\left.+2 \tan ^{-1} x-2 \tan ^{-1} \lambda x\right]
$$

and hence

$$
\bar{D}_{P}=\frac{2}{\pi}\left[\tan ^{-1} x-\tan ^{-1} \lambda x\right]=\frac{2}{\pi} \tan ^{-1} \frac{x-\lambda x}{1+\lambda x^{2}} .
$$

Changing the variable of integration in (10.1) by putting $t=e^{-u}$ gives

$$
\bar{D}_{P}=\max _{x>0} \int_{\log x^{-1}}^{\log x^{-1}+\log \lambda^{-1}} \frac{\log \left(1+e^{2 u}\right)}{\pi e^{u}} d u
$$

and hence

$$
\bar{D}_{P}=\max _{y>0} \int_{y}^{y+\log \lambda^{-1}} \frac{\log \left(1+e^{2 t}\right)}{\pi e^{2 t}} d t
$$

The function

$$
\pi^{-1} x^{-1} \log \left(1+x^{2}\right)
$$

attains its maximum value when $x=1.980291301$, and the maximum value is the constant $C_{P}$ having the value

$$
C_{P}=.2561574434
$$

Hence (10.51) implies that

$$
\bar{D}_{P} \leqq C_{P} \log \lambda^{-1}
$$

and that 


$$
\lim _{\lambda \rightarrow 1} \bar{D}_{P} / \log \lambda^{-1}=C_{P}
$$

In terms of the variable $\theta,(10.55)$ gives

$$
\lim _{\theta \rightarrow 0} \bar{D}_{P} / \theta=C_{P}
$$

11. Universal inequalities. Essentially all of our work has been a study of the manners in which various upper densities are related to $\bar{D}$. The tables show how, for the optimal sequences constructed in $\$ 4$, these various upper densities are related to each other as well as to $\bar{D}$. In particular, Table 4 shows that there exist sequences for which

$$
\bar{D}_{M}^{(5)}<\bar{D}_{M}^{(4)}<\bar{D}_{P}<\bar{D}_{M}^{(3)}<\bar{D}_{M}^{(2)}<\bar{D}_{A}<\bar{D}_{a}=\bar{D}_{M}^{(1)}<\bar{D} .
$$

One service of this fact is to remove the possibility of wasting time in attempts to prove false conjectures to the effect that the inequalities $\bar{D}_{A} \leqq \bar{D}_{M}^{(2)}$, $\bar{D}_{P} \leqq \bar{D}_{M}^{(4)}, \bar{D}_{A} \leqq \bar{D}_{P}$, and others hold universally, that is, for each characteristic sequence of a set of positive integers. If $\bar{D}^{\prime}$ and $\bar{D}^{\prime \prime}$ represent two of the upper densities in (11.1), it is impossible to prove that $\bar{D}^{\prime}<\bar{D}^{\prime \prime}$ holds universally because there exist sequences for which the members of (11.1) are all equal; this happens, for example, whenever the given sequence possesses a density $D$ and whenever $\bar{D}=1$.

It does, however, make sense to recognize that there exist extensive classes of sequences for which the inequalities

$$
\bar{D}_{M}^{(5)} \leqq \bar{D}_{M}^{(4)} \leqq \bar{D}_{P} \leqq \bar{D}_{M}^{(3)} \leqq \bar{D}_{M}^{(2)} \leqq \bar{D}_{A} \leqq \bar{D}_{a}=\bar{D}_{M}^{(1)} \leqq \bar{D}
$$

hold, and to ask which of the inequalities in (11.2) hold universally. The simple and well known inequality

$$
\limsup _{n \rightarrow \infty} \frac{x_{1}+x_{2}+\cdots+x_{n}}{n} \leqq \limsup _{n \rightarrow \infty} x_{n}
$$

and its integral analog imply the universal inequalities

$$
\bar{D}_{M}^{(5)} \leqq \bar{D}_{M}^{(4)} \leqq \bar{D}_{M}^{(3)} \leqq \bar{D}_{M}^{(2)} \leqq \bar{D}_{M}^{(1)} \leqq \bar{D} .
$$

Likewise the well known relation

$$
\limsup _{t \rightarrow 1}(1-t) \sum_{k=1}^{\infty} t^{k} s_{k} \leqq \limsup _{n \rightarrow \infty} \frac{s_{1}+s_{2}+\cdots+s_{n}}{n}
$$

involving the Abel and arithmetic mean transforms implies the universal inequalities $\bar{D}_{a} \leqq \bar{D}$ and $\bar{D}_{A} \leqq \bar{D}_{M}^{(i)}$. We now prove the relatively simple fact that $\bar{D}_{A} \leqq \bar{D}_{a}$. Let $0<r<1$ and let 


$$
F_{A}(r)=(1-r) \sum_{k=1}^{\infty} r^{k}\left(\frac{1}{k} \sum_{j=1}^{k} s_{i}\right), \quad F_{a}(r)=(1-r) \sum_{k=1}^{\infty} r^{k} s_{k}
$$

so that, as (1.5) and (1.7) show,

$$
\bar{D}_{A}=\limsup _{r \rightarrow 1} F_{A}(r), \quad \bar{D}_{a}=\limsup _{r \rightarrow 1} F_{a}(r) .
$$

The fact that, for characteristic sequences, $0 \leqq s_{k} \leqq 1$ justifies the inversions involved in obtaining the relation

$$
\begin{aligned}
F_{A}(r) & =(1-r) \sum_{j=1}^{\infty} s_{j} \sum_{k=j}^{\infty} \frac{r^{k}}{k}=(1-r) \sum_{j=1}^{\infty} s_{j} \sum_{k=j}^{\infty} \int_{0}^{r} t^{k-1} d t \\
& =(1-r) \int_{0}^{r} \frac{1}{(1-t)^{2}}\left\{\frac{1-t}{t} \sum_{j=1}^{\infty} t^{i} s_{j}\right\} d t
\end{aligned}
$$

and hence

$$
F_{A}(r)=(1-r) \int_{0}^{r} \frac{1}{(1-t)^{2}}\left\{\frac{F_{a}(t)}{t}\right\} d t
$$

Use of (11.41) and (11.43) gives

$$
\bar{D}_{A} \leqq \limsup _{t \rightarrow 1}\left\{F_{a}(t) / t\right\}=\bar{D}_{a}
$$

and establishes the result.

Dvoretzky [1, formula (3) which involves misprints] gives the result that $\bar{D}_{P} \leqq \bar{D}_{M}^{(1)}$. That Laplace transforms constitute a principal tool for studies of relations among upper densities is indicated by Dvoretzky [1], and by Zamansky [3] who gives a theory of transformations somewhat similar to those in (3.1) and (3.12). We now show that Laplace transforms can be used to prove the universal inequality $\bar{D}_{P} \leqq \bar{D}_{a}$. Putting the right member of the formula

$$
\frac{1}{1+t^{2}}=t \int_{0}^{\infty} e^{-t y}(1-\cos y) d y
$$

in the formula (2.04) for $\bar{D}_{P}$, and making a change of order of integration which is justified by the fact that the integrands are non-negative, we obtain

$$
\begin{aligned}
\bar{D}_{P} & =\limsup _{x \rightarrow \infty} \frac{2}{\pi} \int_{0}^{\infty}(1-\cos y) d y \int_{0}^{\infty} t e^{-t y} D(x t) d t \\
& =\limsup _{x \rightarrow \infty} \frac{2}{\pi} \int_{0}^{\infty} \frac{1-\cos y}{v^{2}} d y \int_{0}^{\infty} t e^{-t} D\left(\frac{x}{y} t\right) d t .
\end{aligned}
$$

Defining $F_{a}(x)$ by the formula 


$$
F_{a}(x)=\int_{0}^{\infty} t e^{-t} D(x t) d t
$$

in accordance with (2.03), we put (11.51) in the form

$$
\overleftarrow{D}_{P}=\limsup _{x \rightarrow \infty} \frac{2}{\pi} \int_{0}^{\infty} \frac{1-\cos y}{y^{2}} F_{a}\left(\frac{x}{y}\right) d y .
$$

The conclusion $\bar{D}_{P} \leqq \bar{D}_{a}$ then follows. L. A. Rubel, who has made substantial functiontheoretic applications of $\bar{D}_{P}$ and other densities which are mostly not yet published, proved that $\bar{D}_{P} \leqq \bar{D}_{a}$ in a superficially different way by starting with the formula (2.24) for $\bar{D}_{P}$ and using the formula

$$
\log \left(1+\frac{1}{t^{2}}\right)=\int_{0}^{\infty} e^{-t y} \frac{2(1-\cos y)}{y} d y
$$

which can be obtained by dividing (11.5) by $t$ and integrating.

There are 15 cases in which it remains undecided whether inequalities involving upper densities are universally valid. The undecided inequalities involving $\bar{D}_{P}$ are

$$
\bar{D}_{M}^{(5)} \leqq \bar{D}_{P}, \bar{D}_{M}^{(4)} \leqq \bar{D}_{P}, \bar{D}_{P} \leqq \bar{D}_{M}^{(3)}, \bar{D}_{P} \leqq \bar{D}_{M}^{(2)}, \bar{D}_{P} \leqq \bar{D}_{A} .
$$

The undecided inequalities involving $\bar{D}_{A}$ are

$$
\bar{D}_{M}^{(5)} \leqq \bar{D}_{A}, \bar{D}_{M}^{(4)} \leqq \bar{D}_{A}, \bar{D}_{P} \leqq \bar{D}_{A}, \bar{D}_{M}^{(3)} \leqq \bar{D}_{A}, \bar{D}_{M}^{(2)} \leqq \bar{D}_{A} .
$$

The undecided inequalities involving $\bar{D}_{a}$ are

$$
\bar{D}_{M}^{(r)} \leqq \bar{D}_{a}(r \geqq 1), \bar{D}_{a} \leqq \bar{D}_{M}^{(1)} .
$$

As (11.8) indicates, it is undecided whether the equality $\bar{D}_{a}=\bar{D}_{M}^{(1)}$ is universally valid.

\section{REFERENCES}

1. Aryeh Dvoretzky, Sur les suites d'exposants d densitê supêrieure finie, C. R. Acad. Sci. Paris vol. 225 (1947) pp. 481-483.

2. Otto Szász, On products of summability methods, Proc. Amer. Math. Soc. vol. 3 (1952) pp. 257-263.

3. Marc Zamansky, Sur la sommation des séries divergentes, Paris, 1954, 46 pp.

Cornell University,

ITHACA, N. Y. 